\title{
Aplicación del análisis de Fourier al estudio de perfiles de rugosidad de muestras erosionadas
}

\author{
M. Bethencourt ${ }^{(*)}$, F.J. Botana ${ }^{(*)}$, J.J. Calvino ${ }^{(*)}$, M. Marcos ${ }^{(* *)}$ y M.A. Rodríguez-Chacón $\left(^{(*)}\right.$
}

\begin{abstract}
Resumen En este trabajo se propone el análisis de perfiles de rugosidad mediante Transformada de Fourier como herramienta de caracterización de muestras procedentes de ensayos de corrosión-erosión. Los resultados obtenidos indican que este método proporciona información complementaria a la suministrada por parámetros convencionales de rugosidad. El método propuesto puede ser aplicado no sólo en la caracterización de muestras corroídas, sino que es aplicable, en general, a la evaluación de la calidad de acabados superficiales.
\end{abstract}

Palabras clave: Corrosión-erosión. Rugosidad. Transformada de Fourier. Calidad superficial.

\section{Application of Fourier analysis to the study of roughness profiles of eroded samples}

\begin{abstract}
Fourier transforms are applied to analyse surface roughness profiles recorded on samples coming from erosion-corrosion essays. The information retrieved using this method clearly complements that revealed by the more classical roughness amplitude parameters. The analysis procedure here proposed can be applied not only to characterise the surface of corroded samples but, in general, to evaluate the quality of any surface after application of finishing treatments.
\end{abstract}

Keywords: Erosion-corrosion. Roughness. Fourier transform. Surface quality.

\section{INTRODUCCIÓN}

Cuando un material es sometido a un proceso de corrosión-erosión sufre una serie de transformaciones que se traducen en cambios en su textura superficial. Estas modificaciones están relacionadas con el mecanismo a través del cual se ha verificado dicho proceso. Una de las causas que limitan el estudio de los mecanismos de los procesos de corrosión-erosión es la falta de técnicas instrumentales que permitan evaluar cuantitativamente dichos cambios texturales. En el presente trabajo se propone utilizar la técnica de medida de la rugosidad superficial para caracterizar cuantitativamente la topografía de muestras procedentes de ensayos de

(*) Dpto. de Ciencia de los Materiales e Ingeniería Metalúrgica y Química Inorgánica. Univ. de Cádiz. Fac. de Ciencias del Mar. Aptdo. 40. 11510-Puerto Real. (Cádiz, España).E-mail: javier.botana@uca.es

(**) Dpto. de Ingeniería Mecánica y Diseño Industrial. Univ. de Cádiz. Escuela Superior de Ingeniería. c/ Chile s/n. 11003-Cádiz (España). corrosión-erosión. Se ha dedicado especial atención a la aplicación de la Transformada de Fourier en el análisis de los perfiles de rugosidad.

\section{MÉTODO EXPERIMENTAL}

Los perfiles de rugosidad se han obtenido utilizando un rugosímetro Perthometer, modelo $\mathrm{M} 4 \mathrm{Pi} / \mathrm{CNOMO}$. Este equipo permite registrar perfiles de rugosidad con una resolución de hasta $12 \mathrm{~nm}$ y seleccionar distancias de barrido desde $250 \mu \mathrm{m}$ hasta $16 \mathrm{~mm}$.

El estudio se ha realizado sobre muestras de la aleación Al-Mg AA5083 procedentes de ensayos de corrosion-erosión. El dispositivo utilizado, de tipo jet sumergido, y las condiciones de los ensayos se describen en (1). En relación con el dispositivo experimental, hay que señalar que ha sido diseñado en nuestro laboratorio con el objetivo de poder realizar ensayos a alta velocidad, hasta $40 \mathrm{~m} / \mathrm{s}$, utilizando como medio corrosivo una disolución de $\mathrm{NaCl}$ al 3,5\%. 


\section{CONSIDERACIONES TEÓRICAS SOBRE LAS MEDIDAS DE RUGOSIDAD}

La medida de la rugosidad superficial se considera como un medio básico para determinar la calidad de los acabados de las superficies metálicas (2). En las aplicaciones convencionales de la técnica, una vez registrado el perfil de rugosidad es posible calcular toda una serie de parámetros normalizados que proporcionan información cuantitativa de la superficie analizada. En la bibliografía, se describen fundamentalmente dos familias de parámetros: los relacionados con la amplitud del perfil y los relativos a su forma.

Sin duda, los parámetros de amplitud $\left(R_{\mathrm{a}}, R_{\mathrm{z}} \ldots\right)$ son los de uso más extendido, mientras que los de forma se encuentran aún poco implantados. De hecho, hasta muy recientemente estos parámetros se han utilizado a nivel cualitativo y sólo a partir de 1990 se han propuesto métodos cuantitativos para su evaluación (3).

Uno de los métodos recomendados en la Norma DIN 4776 para evaluar la forma de los perfiles, es el basado en las curvas de la tasa de longitud portante del perfil o curvas de Abbott-Firestone. A modo de ejemplo, en la figura 1 se representan dichas curvas para tres superficies con características texturales límite. La primera de ellas (Fig. 1a), corresponde a una superficie que presenta ondulaciones homogéneas; la segunda (Fig. 1b), a una superficie con valles excepcionales de gran profundidad respecto de la superficie media, y la tercera
(Fig. 1c) corresponde a una superficie regular que presenta algunas crestas de gran altitud. La caracterización de superficies reales se puede realizar mediante comparación cualitativa de sus curvas de Abbott con las de los modelos representados en la figura 1.

Un segundo método para evaluar la forma de los perfiles se basa en la obtención de la función de densidad de las amplitudes en el perfil (4), equivalente a la función de densidad de probabilidad utilizada en estadística. A partir de estas curvas, es posible calcular el parámetro denominado sesgo de la distribución $S_{\mathrm{k}}(4)$. En la figura 1 se indica el valor del sesgo correspondiente a cada modelo.

\section{RESULTADOS Y DISCUSIÓN}

En este apartado se incluyen los resultados obtenidos al aplicar la técnica de medida de la rugosidad superficial a la caracterización de muestras procedențes de ensayos de corrosión-erosión. En este punto habría que indicar que esta técnica ha sido escasamente utilizada en la bibliografía en la caracterización de muestras corroidas. En los casos en que ha sido aplicada, su uso se ha restringido al cálculo de algunos parámetros de amplitud (5 y 6).

En la figura 2 se representan los perfiles de rugosidad registrados sobre muestras ensayadas en distintas condiciones experimentales. Concretamente, la muestra M-1 ha sido sometida a la acción de una disolución de $\mathrm{NaCl}$, moviéndose a una

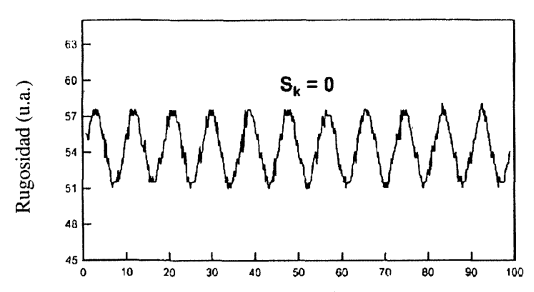

Distancia (u.a.)

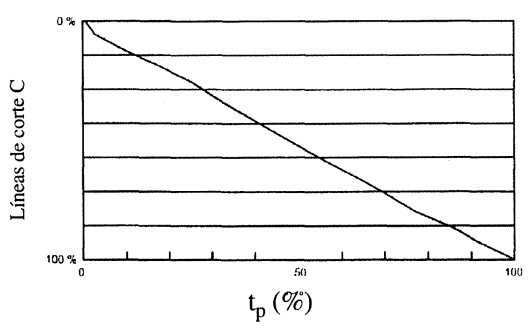

(a)
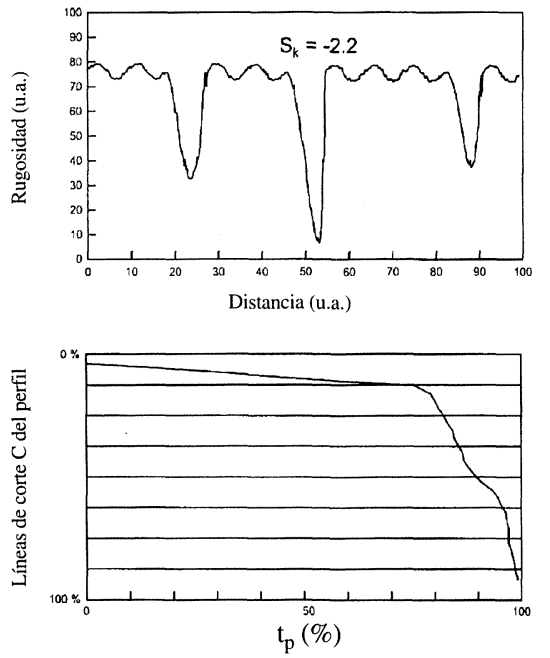

(b)
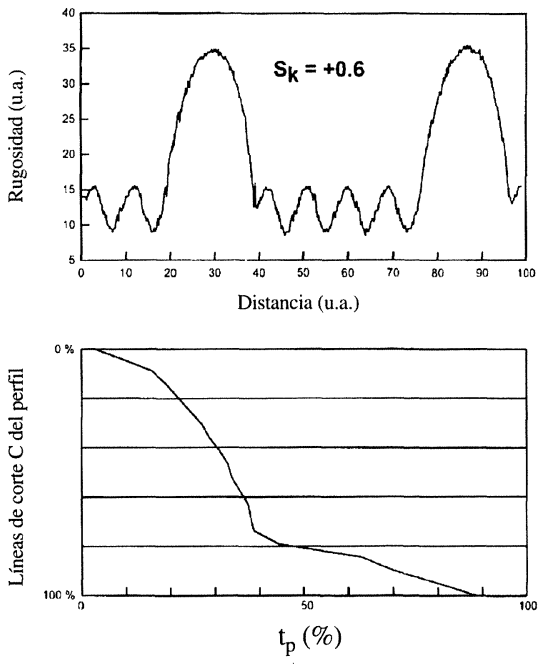

(c)

FIG. 1.- Perfiles de rugosidad y curvas de Abbott-Firestone para tres superficies con características texturales límite. a) Ondulaciones homogéneas; $b$ ) valles excepcionales; $c$ ) crestas excepcionales. Sobre los perfiles de rugosidad se indican los valores del sesgo, $S_{\mathrm{k}}$.

FIG. 1.- Roughness profiles and Abbott-Firestone curves corresponding to three different surface models: a) Homogeneous waves; b) rare valleys; c) rare mountains. Skewness values are indicated on the profiles. 


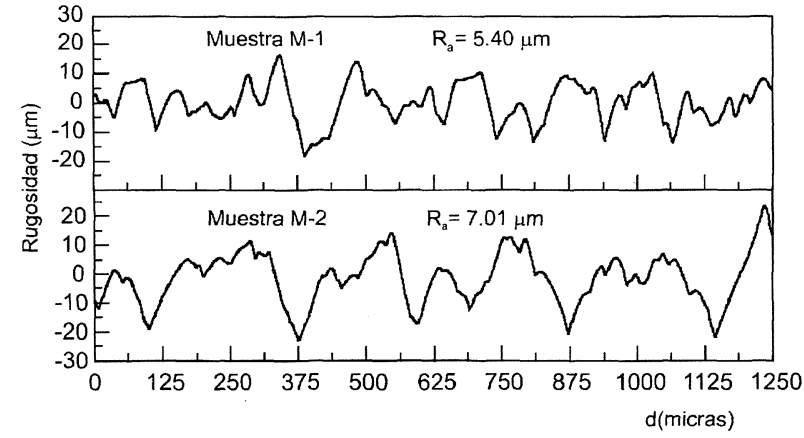

FIG. 2.- Perfiles de rugosidad registrados sobre las zonas erosionadas de las muestras a) M-1 y $b$ ) M-2.

FIG. 2.- Roughness profiles recorded in the eroded zones of a) $M-1$ and b) M-2 samples.

velocidad de $20 \mathrm{~m} / \mathrm{s}$, mientras que la muestra M-2 fue ensayada a $36 \mathrm{~m} / \mathrm{s}$. En esta figura puede observarse que, entre ambos perfiles, existen claras diferencias, tanto de amplitud como de forma. Las diferencias de amplitud existentes entre ambos perfiles se manifiestan a través de los correspondientes valores de $R_{\mathrm{a}}$ que resultaron ser 5,4 y 7,0, respectivamente.

$\mathrm{Al}$ objeto de evaluar las diferencias existentes en las formas de los perfiles, en la figura 3 se representan las curvas de Abbott-Firestone y los valores del sesgo obtenidos a partir de perfiles de rugosidad incluidos en la figura 2 . Los resultados obtenidos ponen de manifiesto que no existen diferencias significativas ni en el aspecto de las curvas de Abbot, ni en los valores calculados para el sesgo. Es decir, utilizando los parámetros que actualmente se proponen en la bibliografía para evaluar la forma de los perfiles de rugosidad no es posible detectar diferencias entre las dos muestras estudiadas. Por tanto, estos resultados vendrían a indicar que el aumento de la velocidad del fluido en los ensayos no causa grandes modificaciones en la textura de las muestras erosionadas.

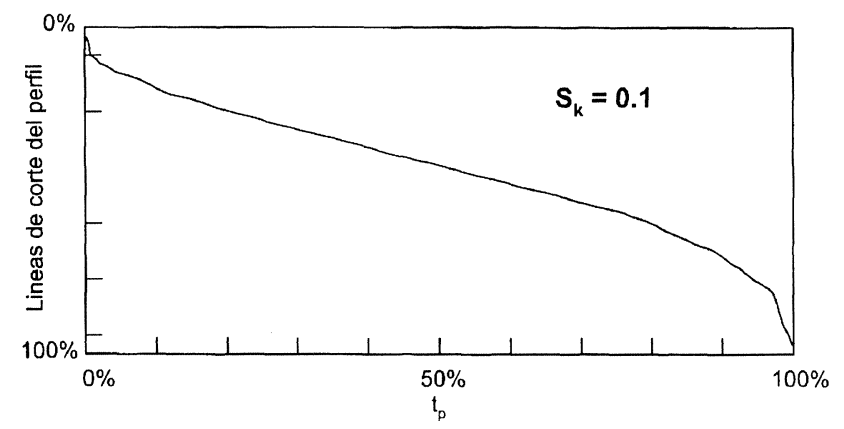

(a) Muestra M-1
Esta conclusión no está de acuerdo con los resultados obtenidos al estudiar las muestras erosionadas mediante microscopía electrónica de barrido (MEB). En la figura 4, se presentan las imágenes MEB adquiridas en las zonas erosionadas de las muestras M-1 y M-2. En ella puede observarse cómo existen claras diferencias texturales entre ambas muestras. Por tanto, estos resultados ponen de manifiesto que existe una cierta insensibilidad de los parámetros de forma convencionales para detectar los cambios que se producen en los ensayos de corrosión erosión.

Con el fin de mejorar la caracterización de las muestras erosionadas, en el presente trabajo se propone realizar un tratamiento de los perfiles de rugosidad mediante Transformadas de Fourier. De esta manera, es posible obtener información cuantitativa complementaria a la que proporcionan los parámetros de amplitud y de forma convencionales.

Los perfiles de rugosidad, como los representados en la figura 2 , pueden tratarse matemáticamente como si fuesen el resultado de la superposición de ondas sinúsoidales con distintas amplitudes, frecuencias y fases. Al realizar la Transformada de Fourier de una señal experimental se obtendrían los valores de las frecuencias de cada una de las ondas armónicas que contribuyen al perfil, junto con el valor de la amplitud y la fase asociada a cada una de ellas (7). Los resultados del análisis del perfil mediante Transformada Rápida de Fourier (FFT) se pueden visualizar con facilidad al representarlos en forma de espectro de potencia (PSD), que es la representación frente al número de onda de la amplitud de cada una de las componentes presentes en el perfil. En la figura 5 se recogen los espectros de potencia obtenidos al aplicar el método a los perfiles experimentales de la figura 2.

En el espectro de potencia correspondiente a la muestra M-1 (Fig. 5a), se observa que las contribuciones de mayor intensidad aparecen centradas

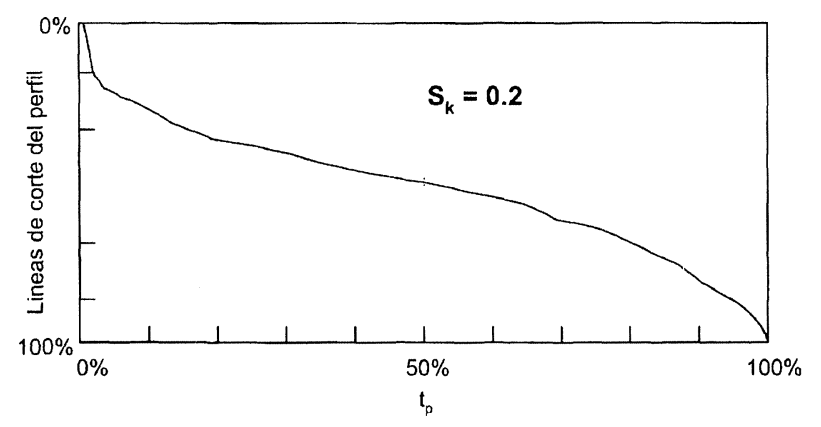

(b) Muestra M-2

FIG. 3.- Curvas de Abbott-Firestone y valores del sesgo calculados a partir de perfiles de rugosidad registrados en las zonas erosionadas de las muestras: a) M-1 y b) M-2.

FIG. 3.- Abbot-Firestone curves and corresponding skewness values calculated from roughness profiles recorded in the eroded zones of a) $M-1$ and b) M-2 samples. 


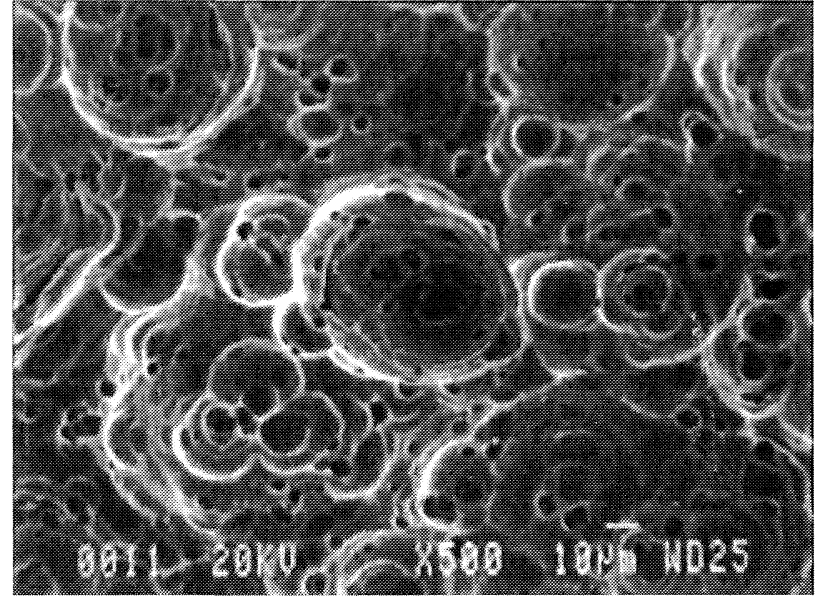

(a) M-1

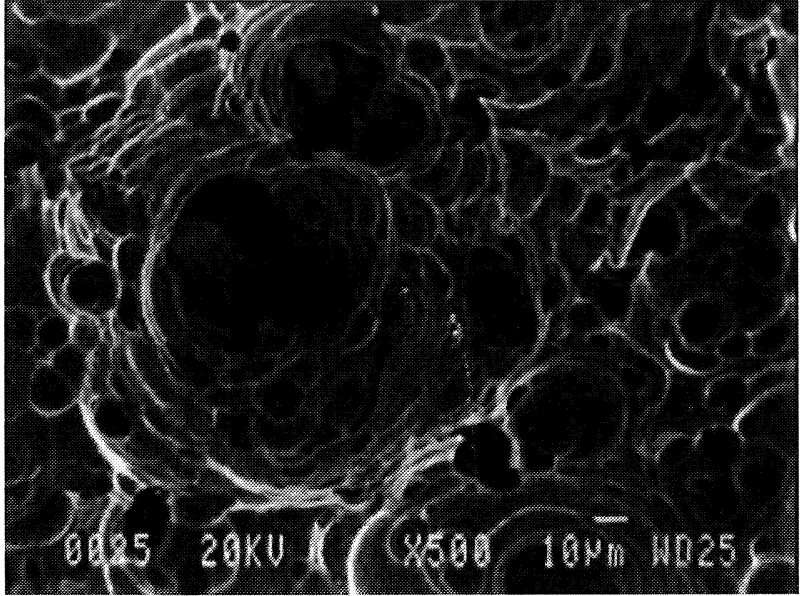

(b) M-2

FIG. 4.- Imágenes MEB adquiridas en las zonas erosionadas de las muestras: $a$ ) M-1 y $b$ ) M-2.

FIG. 4.- SEM images corresponding to the eroded zones of: a) $M-1$ and b) M-2 samples.

a $0,01,0,015$ y $0,02 \mu \mathrm{m}^{-1}$. Esto viene a indicar que el perfil correspondiente a la muestra $\mathrm{M}-1$ puede considerarse formado, mayoritariamente, por la superposición de tres ondas sinusoidales, cuyas amplitudes y números de ondas quedan recogidos en el espectro de potencia.

La principal diferencia entre los PSD de las muestras M-1 y M-2 es que, en la muestra ensayada a alta velocidad (Fig. 5b), se produce un aumento considerable de la amplitud de la modulación de baja frecuencia, centrada a $0,005 \mu \mathrm{m}^{-1}$.

Por tanto, mediante el cálculo de la FFT de los perfiles de rugosidad ha sido posible detectar las diferencias en la textura de las muestras, que se ocasionan como consecuencia del aumento en la

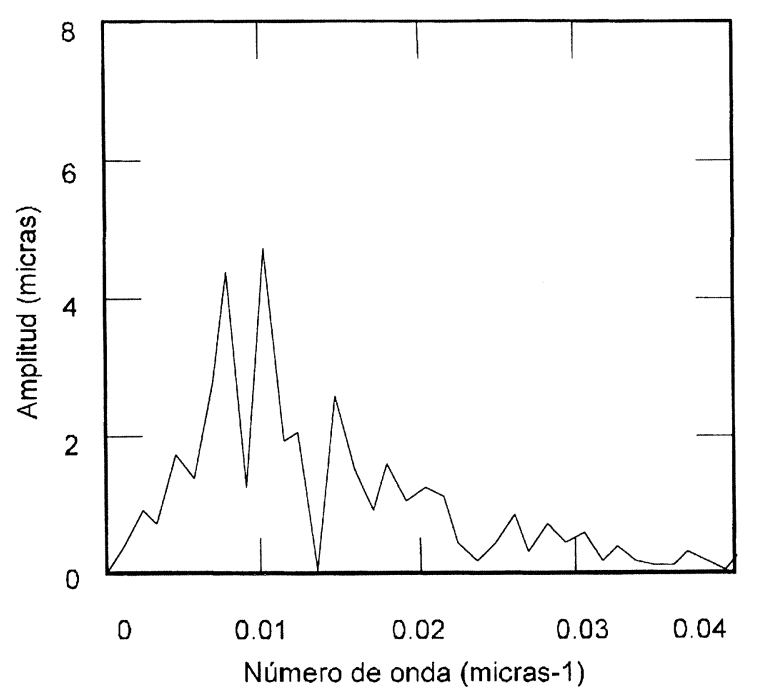

velocidad del fluido. Además, este método presenta la ventaja, frente a otros parámetros de tipo estadístico, de que la información que proporciona es fácilmente correlacionable con la textura de la superficie estudiada.

Como se ha comentado anteriormente, a partir del PSD de la figura $5 \mathrm{~b}$ es posible calcular la amplitud y la fase de la onda centrada a $0,005 \mu \mathrm{m}^{-1}$, que se desarrolla al aumentar la velocidad del fluido en los ensayos de corrosión-erosión. En la figura 6 se representa dicha onda junto con los perfiles de rugosidad de las muestras M-1 y M-2. En esta figura puede comprobarse cómo el perfil de rugosidad de la muestra ensayada a alta velocidad está dominado por la onda de $0,005 \mu \mathrm{m}^{-1}$, mientras que en el perfil de la muestra M-1 no se observa dicha onda.

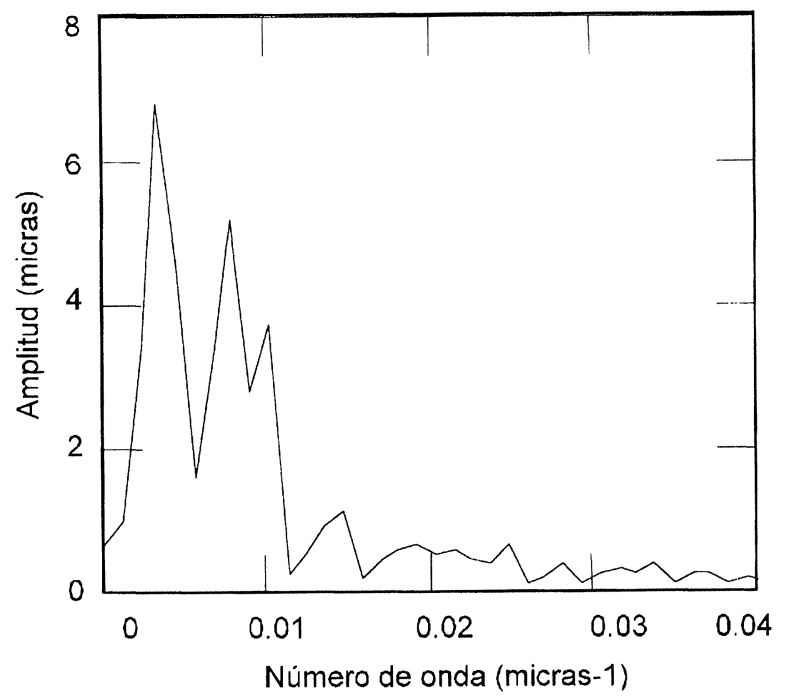

FIG. 5.- Espectros de potencia correspondientes a los perfiles de rugosidad incluidos en la figura 2.

FIG. 5.- PSD corresponding to the roughness profiles shown in Figure 2. 


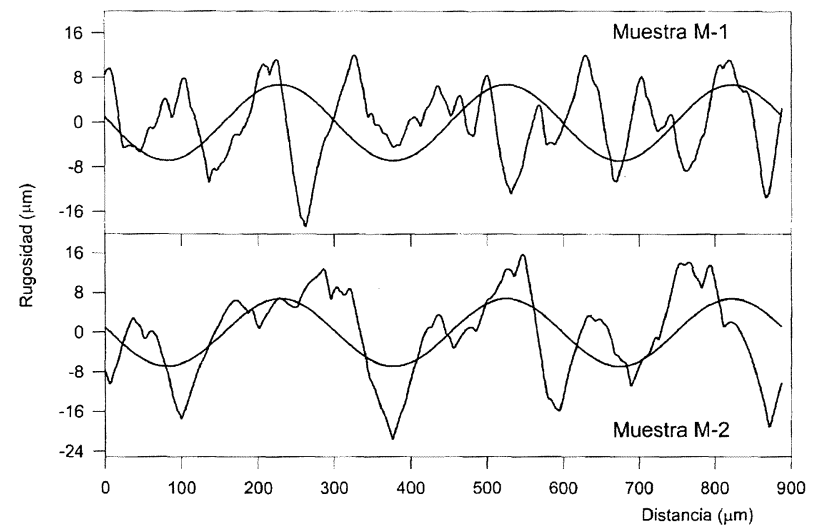

FIG. 6.- Perfiles de rugosidad de las zonas erosionadas de las muestras M-1 y M-2 junto con una onda de tipo seno de frecuencia $0,005 \mu \mathrm{m}^{-1}$.

FIG. 6.- Roughness profiles recorded in the eroded zones of samples $M-1$ and M-2. A sine wave with a frequency of $0.005 \mathrm{\mu m}^{-1}$ has been superimposed on the profiles.

En definitiva, estos resultados parecen indicar que al aumentar la velocidad del medio corrosivo se producen cambios texturales en la superficie de las muestras. Estos cambios se traducen en la aparición de ondulaciones de menor frecuencia que las que se desarrollan en los ensayos realizados a baja velocidad. La longitud de onda de dichas ondulaciones es semejante a las picaduras de mayor diámetro que se observan en la imagen MEB de la figura $4 b$.

\section{CONCLUSIONES}

El estudio efectuado pone de manifiesto que las modificaciones texturales, que, como consecuencia de la variación de la velocidad del fluido, se producen en las muestras erosionadas, pueden evaluarse mediante medidas de rugosidad superficial. No obstante, utilizando los parámetros convencionales sólo se ha logrado detectar los cambios relacionados con la amplitud de los perfiles.

$\mathrm{Al}$ aplicar FFT a señales experimentales ha sido posible identificar la frecuencia, amplitud y fase de cada una de las modulaciones de tipo seno generadas en las superficies como consecuencia de la acción del medio corrosivo. Los resultados obtenidos indican que un aumento de la velocidad del fluido provoca la aparición de ondulaciones de baja frecuencia en la superficie. Inicialmente este tipo de información parece tener interés para profundizar en el conocimiento de los procesos de corrosiónerosión. No obstante, para llegar a establecer correlaciones entre el tipo de modulación, las condiciones de los ensayos y el mecanismo del proceso se hace necesario aplicar el método a un mayor número de muestras ensayadas en diversas condiciones experimentales.

Por último, habría que decir que el análisis de Fourier de señales de rugosidad puede utilizarse para la evaluación de la calidad de distintos acabados superficiales. Los resultados obtenidos en este trabajo ponen de manifiesto que este tipo de análisis proporciona información complementaria a la suministrada por parámetros de forma convencionales.

\section{Agradecimiento}

Este trabajo ha sido financiado por la Comisión Interministerial de Ciencia y Tecnología (CICYT), Proyecto MAR95-2011.

\section{REFERENCIAS}

(1) Bethencourt, M., Botana, F.J., Calvino, J.J., Cauqui, M.A., Marcos, M. y Rodriguez-Chacon, M.A. Proc. $13^{\text {th }}$ Intern. Corros. Congr. Vol. II, paper 198, 1-8 (1996).

(2) Garcia de la Chica, A. Metrología del Acabado Superficial: Rugosidad. Publ. Núm. 35 del Comité de Metrología AECC. Abr. 1993. Madrid (España).

(3) Norma DIN 4776. Mayo 1990.

(4) Norma UNE 82-315-86. Nov. 1986.

(5) Tombinson, W.J. y Matthews, S.J. J. Mat. Sci., 29, 1994: 1.101-1.108.

(6) RAO, B.C.S. y BUCKLEY, D.H. Wear, 105, 1985: 171-182.

(7) Champeney, D.C. Fourier Transforms and their Physical Applications. Academic Press. Londres y Nueva York (EE.UU.) 1973. 